\title{
Assessing the external exposome using wearable passive samplers and high- resolution mass spectrometry among South African children participating in the VHEMBE study
}

Jeremy P. Koelmel ${ }^{1}$, Elizabeth Z. Lin ${ }^{1}$, Kayley DeLay ${ }^{1}$, Antony J. Williams ${ }^{2}$, Yakun Zhou ${ }^{1}$, Riana Bornman ${ }^{3}$, Muvhulawa Obida ${ }^{3}$, Jonathan Chevrier ${ }^{4}$, Krystal J. Godri Pollitt ${ }^{*}$

${ }^{1}$ Department of Environmental Health Sciences, Yale School of Public Health, New Haven, CT, 06520, United States

2 Center for Computational Toxicology and Exposure, Office of Research and Development, United States Environmental Protection Agency, Research Triangle Park, NC, 27711, United States

${ }^{3}$ University of Pretoria Institute for Sustainable Malaria Control and School of Health Systems and Public Health, University of Pretoria, Pretoria, 0028, South Africa

${ }^{4}$ Department of Epidemiology, Biostatistics and Occupational Health, Faculty of Medicine, McGill University, Montréal, QC, H3A 1A2, Canada. 

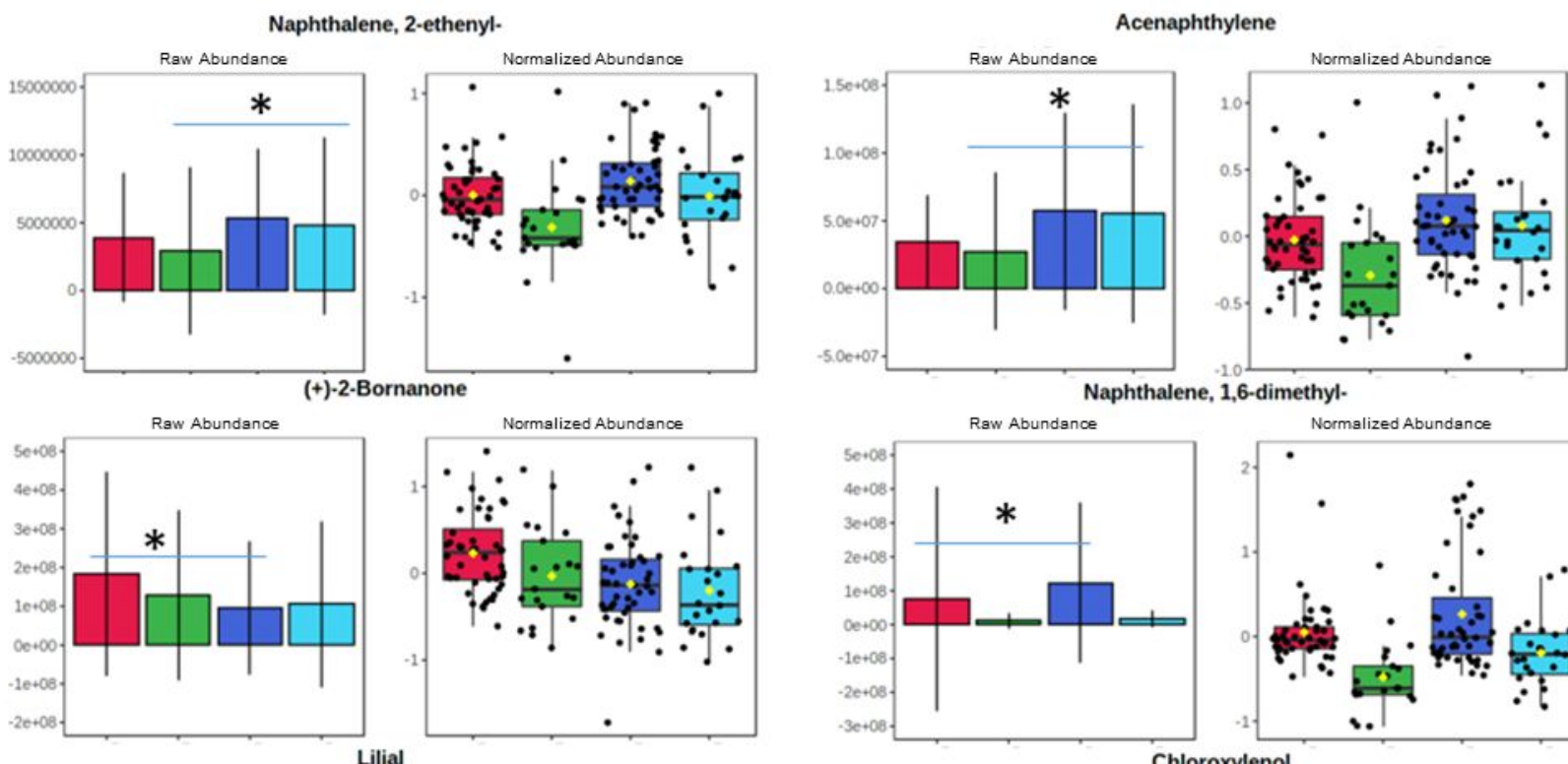

Naphthalene, 1,6-dimethyl-
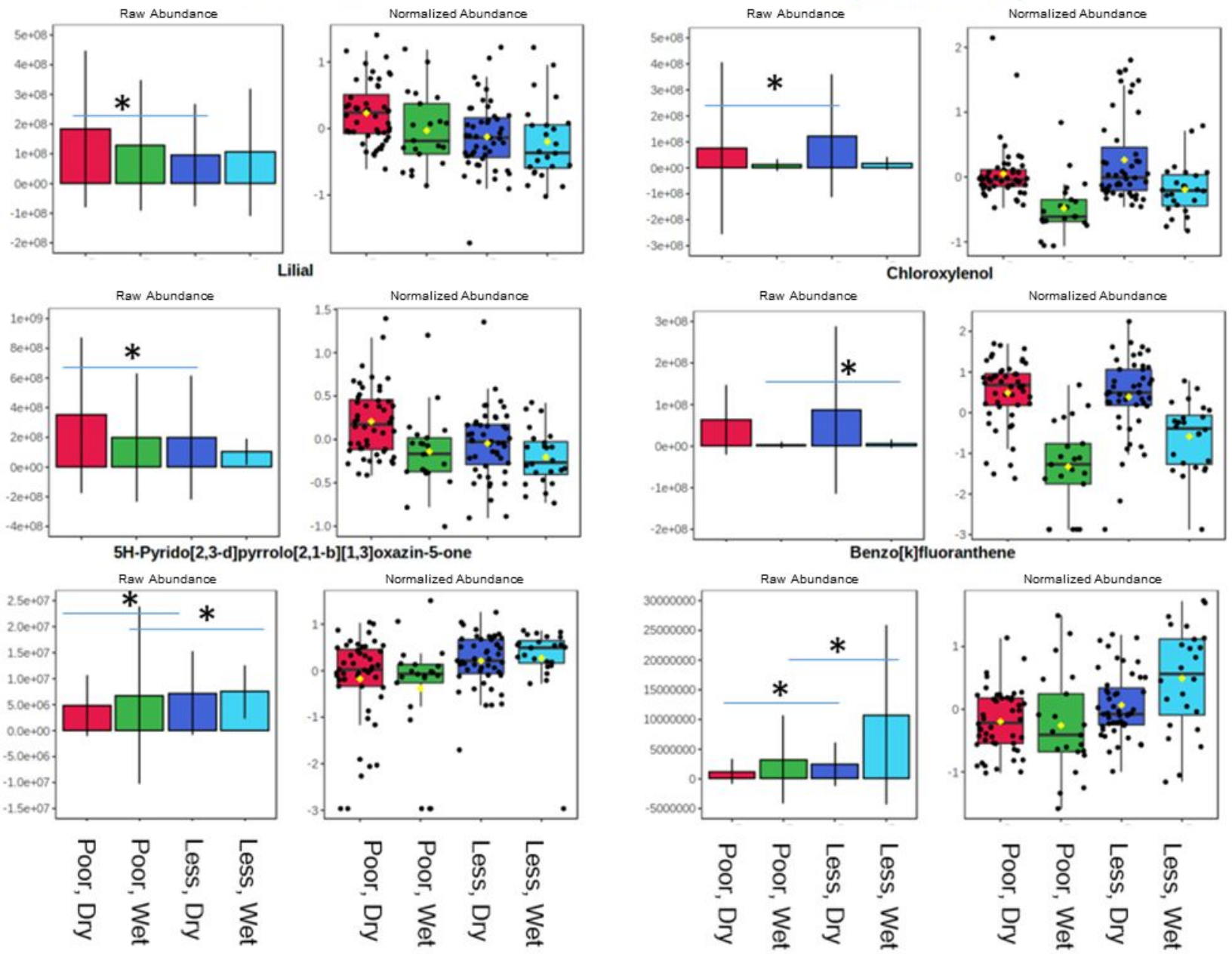

Figure S1: A subset of chemicals and their significance across poverty level (more poor: "poor", less poor: "less"), grouped by season (wet season: "wet", dry season: "dry"). Significance is based on a post-hoc Fisher's LSD test and ANOVA with Hochberg correction (adjusted pvalue). Significant comparisons between poor and less poor are shown. Raw and normalized (log-scaled and mean centered) abundance data are shown, with statistics performed on the normalized data. 


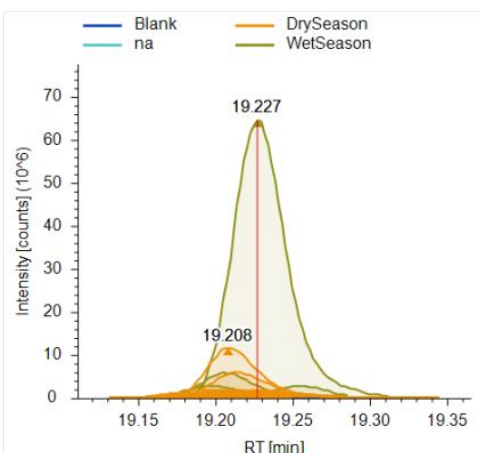

Structure Name Caffeine
Total Score 96.5

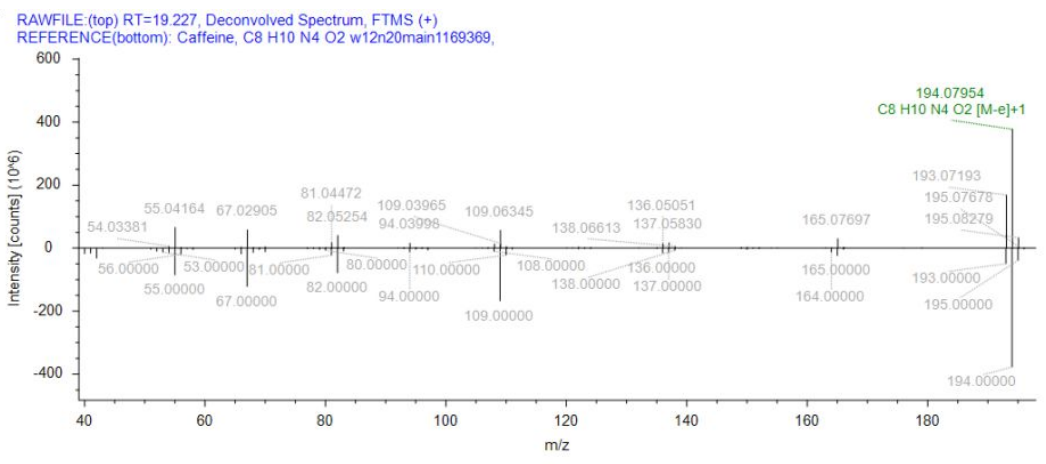

HRF Score

99.4

\section{SI}

835
$\Delta$ Mass [ppm]
-1.46
RI Delta

8

42 Figure S2. Spectral evidence used in suspect screening to identify caffeine. Note that a Level 2 (High Confidence) assignment was provided when RI delta $<50, R S I>600$, and RHRF $>75$. In compounds of interest manual review was done to show that no other compounds in the library had a similar spectra and matching $R I$, and that other evidence including observation of molecular ion, isotopic ratios, peak shape, and database occurrence. For caffeine, all these lines of evidence were observed (molecular ion exact mass match, high El and high-resolution mass filter score, retention index match, and quality peak shape higher than background signal). Given the caffeine is relative non-volatile, we expect the detected chemical was associated with deposited particulate. It is also useful to note the heterogeneity of exposures ranging across several magnitudes between participants. 